\title{
RESEARCH
}

Open Access

\section{Sirt6 inhibition delays the onset of experimental autoimmune encephalomyelitis by reducing dendritic cell migration}

Giovanni Ferrara $^{1 * \dagger}$ (D) Andrea Benzi ${ }^{2 \dagger}$, Laura Sturla ${ }^{2}$, Daniela Marubbi ${ }^{1,2}$, Davide Frumento ${ }^{2}$, Sonia Spinelli ${ }^{2}$, Elena Abbotto ${ }^{2}$, Federico Ivaldi ${ }^{3}$, Maria von Holtey ${ }^{4}$, Maximilien Murone ${ }^{5}$, Alessio Nencioni ${ }^{1,6}$,

Antonio Uccelli ${ }^{1,3}$ and Santina Bruzzone ${ }^{2}$

\begin{abstract}
Background: Experimental autoimmune encephalomyelitis (EAE) is the most common animal model of multiple sclerosis (MS), a neuroinflammatory and demyelinating disease characterized by multifocal perivascular infiltrates of immune cells. Although EAE is predominantly considered a T helper 1-driven autoimmune disease, mounting evidence suggests that activated dendritic cells (DC), which are the bridge between innate and adaptive immunity, also contribute to its pathogenesis. Sirtuin 6 (SIRT6), a NAD -dependent deacetylase involved in genome maintenance and in metabolic homeostasis, regulates DC activation, and its pharmacological inhibition could, therefore, play a role in EAE development.

Methods: EAE was induced in female C57b//6 mice by MOG35-55 injection. The effect of treatment with a small compound SIRT6 inhibitor, administered according to therapeutic and preventive protocols, was assessed by evaluating the clinical EAE score. SIRT6 inhibition was confirmed by Western blot analysis by assessing the acetylation of histone 3 lysine 9, a known SIRT6 substrate. The expression of DC activation and migration markers was evaluated by FACS in mouse lymph nodes. In addition, the expression of inflammatory and anti-inflammatory cytokines in the spinal cord were assessed by qPCR. T cell infiltration in spinal cords was evaluated by immunofluorescence imaging. The effect of Sirt6 inhibition on the migration of resting and activated bone marrow-derived dendritic cells was investigated in in vitro chemotaxis assays.

Results: Preventive pharmacological Sirt6 inhibition effectively delayed EAE disease onset through a novel regulatory mechanism, i.e., by reducing the representation of CXCR4-positive and of CXCR4/CCR7-double-positive DC in lymph nodes. The delay in EAE onset correlated with the early downregulation in the expression of CD40 on activated lymph node DC, with increased level of the anti-inflammatory cytokine IL-10, and with a reduced encephalitogenic T cell infiltration in the central nervous system. Consistent with the in vivo data, in vitro pharmacological Sirt6 inhibition in LPS-stimulated, bone marrow-derived DC reduced CCL19/CCL21- and SDF-1-induced DC migration.

(Continued on next page)
\end{abstract}

\footnotetext{
* Correspondence: Giovanni.Ferrara@hsanmartino.it

${ }^{\dagger}$ Giovanni Ferrara and Andrea Benzi contributed equally to this work.

'Ospedale Policlinico San Martino, IRCCS, Largo R. Benzi, 10, 16132 Genova, Italy

Full list of author information is available at the end of the article
}

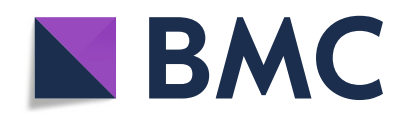

( ) The Author(s). 2020 Open Access This article is licensed under a Creative Commons Attribution 4.0 International License, which permits use, sharing, adaptation, distribution and reproduction in any medium or format, as long as you give appropriate credit to the original author(s) and the source, provide a link to the Creative Commons licence, and indicate if changes were made. The images or other third party material in this article are included in the article's Creative Commons licence, unless indicated otherwise in a credit line to the material. If material is not included in the article's Creative Commons licence and your intended use is not permitted by statutory regulation or exceeds the permitted use, you will need to obtain permission directly from the copyright holder. To view a copy of this licence, visit http://creativecommons.org/licenses/by/4.0/. The Creative Commons Public Domain Dedication waiver (http://creativecommons.org/publicdomain/zero/1.0/) applies to the data made available in this article, unless otherwise stated in a credit line to the data. 
(Continued from previous page)

Conclusions: Our findings indicate the ability of Sirt6 inhibition to impair DC migration, to downregulate pathogenic T cell

inflammatory responses and to delay EAE onset. Therefore, Sirt6 might represent a valuable target for developing novel

therapeutic agents for the treatment of early stages of MS, or of other autoimmune disorders.

Keywords: SIRT6, EAE, Dendritic cells, Migration, Clinically isolated syndrome, MS

\section{Introduction}

Sirtuin 6 (SIRT6) belongs to the sirtuin family of proteins, which includes seven members (SIRT1-7). Sirtuins are enzymes that remove acetyl and acyl groups from target proteins by using $\mathrm{NAD}^{+}$as co-substrate [1]. SIRT6 has important roles in physiological and pathological processes, regulating aging, cancer, obesity, insulin resistance, inflammation, and energy metabolism [1]. Regarding immunity and inflammation, our group and others demonstrated that SIRT6 promotes the release of TNF $\alpha$ through its deacetylase activity [2, 3]. Moreover, SIRT6-mediated deacylation of TNF $\alpha$ was reported to enhance the release of this cytokine [4]. In addition to TNF $\alpha$, SIRT6 also regulates the secretion of other proinflammatory cytokines, such as IFN $\gamma$ and IL8 $[3,5,6]$ through the activation of the cation channel TRPM2 [5]. SIRT6 also plays an important role in T lymphocyte biology, since Sirt6KO mice develop lymphopenia [7] and the availability of $\mathrm{NAD}^{+}$for SIRT6 activity is pivotal for the regulation of $\mathrm{T}$ cell metabolism during the early and late stages of acute inflammation [8]. SIRT6 also promotes differentiation and maturation of dendritic cells (DC; ref. [9]), which are antigen-presenting cells involved in the initiation of adaptive immune responses. Sirt6KO mice have a reduced representation of DC precursors in their bone marrow; DC from Sirt6KO mice express lower levels of class II MHC, of costimulatory molecules, and of the chemokine receptor CCR7 and are less immunostimulatory than wild-type DC [9].

The lack of SIRT6 inhibitors has so far hampered the study of the effect pharmacological SIRT6 inhibition in animal models. We identified the first small molecules SIRT6 inhibitors, by structure-based in silico compound screens [10]. The use of these inhibitors in in vitro cell systems replicated the biological effects that would be predicted based on SIRT6 role in different cell functions. Our inhibitors reduced TNF $\alpha$ production by phorbol myristate acetate-stimulated cancer cells [10] and by peripheral blood mononuclear cells (PBMC) stimulated with phytohemagglutinin (PHA) or with allogeneic antigenpresenting cells [11]. In addition, they reduced $T$ cell proliferation in response to staphylococcal enterotoxin $\mathrm{B}$ (SEB) and to PHA [12]. In line with biological effects regulated by SIRT6, the SIRT6 inhibitors we identified also increased glucose uptake by muscle cells and potentiated the anticancer effects of other chemotherapeutic drugs in cancer cells [10-13]. In addition, a SIRT6 inhibitor with quinazolinedione structure (named $\mathbf{1}$ ) was administered to mice in an in vivo study of SIRT6 inhibition for treating type 2 diabetes [14]. Here, the administration of $1 \mathrm{im}$ proved glucose tolerance and other metabolic parameters, such as insulin resistance as well as triglycerides and cholesterol levels [14]. Importantly, 1 was well tolerated with no evidence of clinical toxicity [14].

Based on these studies, here we used the same small molecule SIRT6 inhibitor (1) to explore the role of SIRT6 in the development of experimental autoimmune encephalomyelitis (EAE), a prototypical mouse model of autoimmune disorder.

\section{Materials and methods \\ Materials}

The SIRT6 inhibitor 2,4-dioxo- $N$-(4-(pyridin-3 yloxy)phenyl)-1,2,3,4-tetrahydroquinazoline-6-sulfonamide (herein named 1) was obtained from Enamine (Riga, Latvia).

\section{PK study}

Compound 1 was dissolved in 10\% kleptose in water at $0.5 \mathrm{mg} / \mathrm{mL}$ and $\mathrm{pH}$ was adjusted to 4 using $1 \mathrm{~N} \mathrm{HCl}$. Mice were injected intraperitoneally with $5 \mathrm{mg} / \mathrm{kg}$ body weight of 1 . The PK study was carried out as in [12], was approved by the Ethics Committee IACUC (institutional animal care and use committee), and was performed by the company (CRO) Medicilon.

\section{EAE Induction}

Chronic EAE was induced in female C57BL/6 J mice (68 weeks of age, weighing $18.5 \pm 1.5 \mathrm{~g}$ ) by subcutaneous injection at two different sites in the right and left flanks with an emulsion ( $200 \mu \mathrm{l}$ total) containing $200 \mu \mathrm{g}$ myelin oligodendrocyte glycoprotein peptide spanning amino acids 35-55 (MOG35-55) (Espikem) in incomplete Freund's adjuvant (Sigma-Aldrich) supplemented with $600 \mu \mathrm{g}$ Mycobacterium tuberculosis (strain H37RA; Difco). Mice were injected in the tail vein with $400 \mathrm{ng}$ pertussis toxin (Sigma-Aldrich) in $100 \mu \mathrm{l}$ of phosphate buffer saline solution (PBS, $\mathrm{pH} 7.6$ ) immediately and 48 $\mathrm{h}$ after the immunization. The mice were scored daily for clinical manifestations of EAE on a scale of 0-5 [15].

All applicable international, national, and/or institutional guidelines for the care and use of animals were followed (Decreto Legislativo 4 March 2014, n. 26, 
legislative transposition of Directive 2010/63/EU of the European Parliament and of the Council of 22 September 2010 on the protection of animals used for scientific purposes). The research protocol was approved by the Ethical Committee for Animal Experimentation of the IRCCS San Martino hospital, Genoa, Italy, and by the Italian Ministry of Health (project nr, 553/2018-PR).

\section{Treatment protocol with the SIRT6 inhibitor}

The effects of the SIRT6 inhibitor on the severity of EAE was assessed considering two different protocols: 1 was administered following a "preventive" protocol (i.e., administration started at day 0), or following a "therapeutic" protocol (i.e., administration started at disease onset). Mice were randomized in the different experimental groups (11 animals/group). Compound 1 was intraperitoneally injected at two different doses: $30 \mathrm{mg} / \mathrm{kg}$ once or twice/day. All the immunized animals developed EAE. Mice were sacrificed at different time points: 7 or 9 days post immunization (dpi), at disease onset in vehicle-treated mice (i.e., at 11-14 dpi) or at 4 days post onset (dpo, i.e., at 15-17 dpi). At sacrifice, blood was immediately collected, transferred into $1.5-\mathrm{ml}$ EDTA coated tubes (Sarstedt), and centrifuged to obtain plasma. Spleen and lymph nodes were collected; cells were dissociated, counted, and subjected to FACS analysis to evaluate DC number (see below). Spinal cords were also collected and immediately frozen or fixed with $4 \%$ paraformaldehyde.

\section{FACS analysis}

Cells (collected from spleen and lymph node from 1and vehicle-treated mice, see above) were resuspended in $100 \mu \mathrm{l}$ of FACS buffer (PBS, pH 7.2, containing 0.5\% bovine serum albumin) and stained with appropriate conjugated antibodies for $30 \mathrm{~min}$ at $4{ }^{\circ} \mathrm{C}$. To assess the migratory markers expression on DC, lymph nodes were collected at $7 \mathrm{dpi}$ and disease onset (11 dpi) and passed through a $70-\mu \mathrm{m}$ nylon cell strainer (Falcon) to prepare a single-cell suspension and used to optimize staining conditions. Lymph node cells were counted automatically (Countess, Invitrogen), and BV510positive (Becton-Dickinson) DC (live cells) were gated APC Vio 770-conjugated anti-CD45, Pe Vio770-conjugated anti-MHCII, and APC-conjugated anti-CD11c antibodies (diluted 1:100, Miltenyi) (see Additional file 1) and antibodies against relevant surface markers, PE-conjugated anti-CCR7, FITCconjugated anti-CXCR4 (diluted 1:100, Miltenyi), PBconjugated anti-CD40, or PerCP-conjugated antiCD86 (diluted 1:100, Biolegend). Data were acquired on a FACS Canto II (Becton-Dickinson) and analyzed using DIVA 6.1 software.

\section{TNFa determination in plasma}

TNF $\alpha$ was evaluated with a commercial ELISA kit (Biolegend, San Diego, CA).

\section{Immunofluorescence}

Spinal cords were fixed overnight in $4 \%$ paraformaldehyde in PBS, cryoprotected overnight in $20 \%$ sucrose, embedded in Tissue Tek O.C.T., and frozen at $-80^{\circ} \mathrm{C}$. Sections were obtained with a Leica CM3050 S cryostat and permeabilized with $0.1 \%$ Triton X-100 (Bio-Rad) in PBS for 30 min. The antibody against CD4 (rat monoclonal antimouse CD4 RM4-5 clone (1:100, 550280 BD Pharmingen) was incubated over-night at $4{ }^{\circ} \mathrm{C}$ in PBS with $10 \%$ goat serum (Sigma-Aldrich) and 0.1\% BSA (Sigma-Aldrich). Next, the secondary antibody (goat anti-rat Cy3-conjugated AffiniPure (1:500, 112-165-167 Jackson Immunoresearch Laboratories), was incubated $1 \mathrm{~h}$ at room temperature in PBS containing $10 \%$ goat serum. Nuclei were stained with Hoechst $33342(1 \mu \mathrm{g} / \mathrm{ml}$, Sigma-Aldrich).

\section{Western blot analysis}

Splenocytes were isolated from vehicle and 1-treated animals at 7 dpi. Primary bone-marrow-derived dendritic cells (BMDDC) were obtained as described below and treated (or not) for $18 \mathrm{~h}$ with $1(50 \mu \mathrm{M}$, final concentration). Splenocytes and DC were lysed in $50 \mathrm{mM}$ Tris$\mathrm{HCl}$ (pH 8), $150 \mathrm{mM} \mathrm{NaCl}, 1 \mathrm{mM}$ EDTA, $1 \mathrm{mM} \mathrm{NaF}, 10$ $\mathrm{mM}$ trichostatin A, $10 \mathrm{mM}$ nicotinamide, $0.5 \mathrm{mM}$ DTT, and protease inhibitor cocktail. Lysates ( $30 \mu \mathrm{g}$ proteins) were loaded on a $10 \%$ polyacrylamide gel and separated by SDS-PAGE, and proteins were transferred to nitrocellulose membranes. Detection was performed with the following primary antibodies: anti-acetylated H3K9 (rabbit polyclonal; Abcam) or anti-vinculin (rabbit monoclonal, Cell Signaling Technology, Danvers, MA). Following incubation with the appropriate secondary antibodies and ECL detection (GE Healthcare, Milan, Italy), band intensity was quantified with the ChemiDoc imaging system (Bio-Rad, Milan, Italy).

\section{qPCR analyses}

RNA extraction from spinal cord was performed using QIAzol Lysis Reagent and TissueLyser instrument (Qiagen); the homogenates were extracted with chloroform, and then RNA was purified using RNeasy Mini Kit (Qiagen) and quantified using a NanoDrop spectrophotometer (Nanodrop Technologies, Wilmington, DE). The cDNA was synthesized by using iScript cDNASynthesis Kit (Bio-Rad Laboratories, Milan, Italy) starting from $1 \mu \mathrm{g}$ of total RNA. Each sample was assayed in triplicate in a 10- $\mu$ l amplification reaction, containing $30 \mathrm{ng}$ of cDNA, $0.4 \mu \mathrm{M}$ of sense and antisense primers, and $10 \mu \mathrm{l}$ of $2 X$ iQ SYBR Green Supermix Sample (Bio-Rad). The amplification program included 40 cycles of two steps, 
each cycle including heating to 95 and $60^{\circ} \mathrm{C}$, respectively. To verify the purity of the products, a melting curve was produced after each run. PCR-specific primers were designed through Beacon Designer 2.0 Software (Bio-Rad Laboratories) and were the following: IFN $\gamma, 5^{\prime}$-GGAGGA ACTGGCAAAAGGAT-3' (forward) and 5' -TTCAAGAC TTCAAAGAGTCTGAGG-3' (reverse); IL12, 5'-CCAG GTGTCTTAGCCAGTCC-3' (forward) and 5'-GCAGTG CAGGAATAATGTTTCA-3' (reverse); IL10, 5' -TAAG GCTGGCCACACTTGAG-3' (forward) and 5'-GTTT TCAGGGATGAAGCGGC-3' (reverse). Values were normalized to murine TBP (TATA Binding Protein) and HPRT1 (Hypoxanthine Phosphoribosyltransferase 1) and $\beta$-actin mRNA expression measured using the following specific primers: TBP, $5^{\prime}$-GAAGCTGCGGTACAATTC CAG-3' (forward) and 5'-CCCCTTGACCCTTCACCA AT-3' (reverse); HPRT1, 5'-CCCTGGTTAAGCAGTA CAGCCCC-3' (forward) and 5'AGTCTGGCCTGTATCCAACACTTCG-3' (everse); $\beta$-actin, (5' -GGCACCACAC CTTCTACAATGAG-3' (forward) and 5'-GACCAGAG GCATACAGGGACAG-3' (reverse). Quantitative realtime PCR (qPCR) was performed in an iQ5 real-time PCR detection system (Bio-Rad Laboratories). Statistical analysis of the qPCR was performed using the iQ5 Optical System Software version 1.0 (Bio-Rad Laboratories) based on the $2^{-\Delta \Delta C t}$ method [16], which calculated the relative change in expression of the target genes, normalized to TBP, HPRT1 and $\beta$-actin. The dissociation curve for each amplification was analyzed to confirm absence of unspecific PCR products.

\section{Generation of bone-marrow-derived dendritic cells}

Primary bone-marrow-derived dendritic cells (BMDDC) were obtained as described previously [15]. Briefly, at day 0 , bone-marrow cells from mice were flushed from the femur and tibia and passed through a 70- $\mu \mathrm{m}$ nylon cell strainer (Falcon). The cell suspension was seeded in the presence of granulocyte macrophage colonystimulating factor $(20 \mathrm{ng} / \mathrm{ml}$, Miltenyi) and interleukin 4 (10 ng/ml, Biolegend). After 7 days, cells were analyzed by FACS for surface marker CD11c. Purity of CD11c ${ }^{+}$ cells assessed by FACS with APC-conjugated antiCD11c antibody (Becton-Dickinson) was at least 90\%.

\section{Dendritic cell migration}

DC were treated (or not) for $18 \mathrm{~h}$ with $1(50 \mu \mathrm{M}$, final concentration) and/or with LPS $(0.5 \mu \mathrm{g} / \mathrm{ml}$, final concentration). DC were resuspended at $8 \times 10^{5}$ cells $/ \mathrm{ml}$ in chemotaxis buffer (HBSS, PBS, and 5\% albumin, 39:16:1). Chemotaxis assays were performed using 96-well ChemoTx system microplates (Neuro Probe, Gaithersburg, MD) with a $5-\mu \mathrm{m}$ pore size polycarbonate filter. Filters were pre-coated with superfibronectin $(1 \mu \mathrm{g} / \mathrm{ml})$. CCL19 and CCL21 $(500 \mathrm{ng} / \mathrm{ml})$ or SDF-1 $(300 \mathrm{ng} / \mathrm{ml})$ were added (or not) to the bottom wells. Cell suspensions $(25 \mu \mathrm{l})$ were then placed on top of the filter and allowed to migrate for $3 \mathrm{~h}$ at $37^{\circ} \mathrm{C}$. Counting of transmigrated cells was performed as described previously [17]. Results were expressed as chemotaxis index (number of cells that migrated toward chemoattractant/number of cells that migrated toward medium).

\section{Results \\ SIRT6 inhibition delays EAE onset}

Given the role for SIRT6 in lymphocyte and dendritic cell activation $[3,9]$, we evaluated the effect of the SIRT6 inhibitor, compound $\mathbf{1}$ in a Th1/Th17-driven model of autoimmune disorder of the central nervous system (CNS), such as EAE. In order to investigate the effect of Sirt6 inhibition during the inflammatory phase and to provide the experimental basis for a possible, new therapeutic approach in MS, $\mathbf{1}$ was administered according to both a "preventive" (i.e., administration before disease onset) and a "therapeutic" (i.e., administration after disease onset) protocol. 1 was administered at the dosage of $30 \mathrm{mg} / \mathrm{kg}$ body weight once a day, starting on the day of mouse immunization with MOG35-55 (preventive treatment) or at the moment of disease onset (therapeutic treatment).

As shown in the Fig. 1a, in the preventive protocol, mouse treatment with 1 resulted in a reduction in the neurological impairment (Fig. 1a), and the effect was statistically significant throughout the period between disease onset and day 14 post immunization (14 dpi; Fig. 1b). However, no significant effect of preventive treatment with 1 between day 14 and day 28 was observed (Fig. 1c). When administered according to our therapeutic protocol, 1 failed to show effects on the disease score.

Given the short half-life of $\mathbf{1}\left(t_{1 / 2}=0.95 \mathrm{~h}\right)$, a new administration protocol in the preventive treatment was tested, according to which the compound was administered twice daily, starting from the day of immunization. With this schedule, the effect of $\mathbf{1}$ on disease onset was more pronounced (Fig. 1d, e), with 1 markedly delaying disease onset (Fig. 1f) as calculated by Kaplan-Meier analysis.

When administered twice a day according to the therapeutic protocol, 1 failed to show an impact on disease course, especially in the chronic phase (Fig. 1g-i).

$\mathbf{1}$ did not show renal or liver toxicity, nor did $\mathbf{1}$-treated mice show weight loss, increased frequency of infections, or other clinically detectable adverse events (data not shown and ref. [14]).

\section{SIRT6 inhibition affects immune cell responses during EAE development}

In the attempt to identify the mechanism for the observed delay in EAE onset in the animals treated with $\mathbf{1}$ according to the preventive protocol (administration of 1 twice a day), we analyzed numerous parameters in 


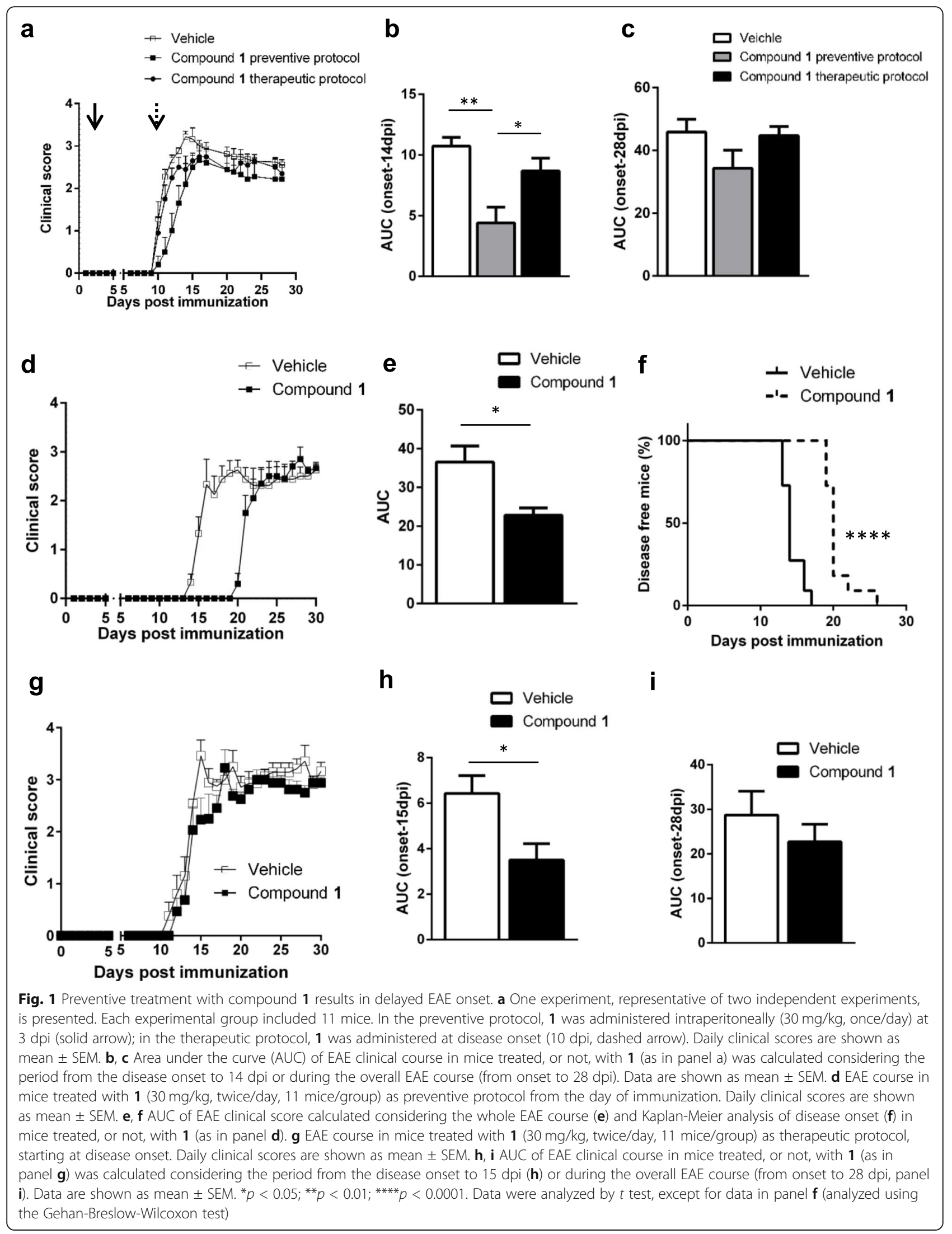


immune cells that were isolated from spleen and lymph nodes in animals sacrificed on day 7 or on day 9 post immunization (7 dpi, $9 \mathrm{dpi}$ ), the latter being two crucial time points of the inflammatory phase, as well as on the day of EAE onset (defined as the moment EAE became clinically detectable in our mice from the control cohort, i.e., 15-17 dpi). Western blot analyses performed on splenocytes obtained at $7 \mathrm{dpi}$ showed that $\mathbf{1}$ significantly increased the acetylation of histone 3 lysine 9 (H3K9), a known substrate of SIRT6 deacetylase activity (Fig. 2a), thus confirming that i.p. 1 injection reduced SIRT6 enzymatic activity in immune cells in vivo.

As compared to vehicle-treated mice, the total numbers of spleen and of lymph node cells were significantly reduced in animals treated with $\mathbf{1}$ at 7 and 9 dpi and at disease onset (Fig. 2b, c). Lymphocytes infiltrating the spinal cord were quantified in vehicle- and in 1-treated mice at 15-17 dpi by immunofluorescence staining of $\mathrm{CD} 4^{+}$cells. 1-treated mice exhibited a significantly lower infiltration with $\mathrm{CD}_{4}^{+}$cells as compared to the control animals, in agreement with the observed difference in clinical score observed at the same time point (Fig. 2d).

\section{Compound 1 administration reduces the ability of CXCR4 ${ }^{+}$DC to migrate to the afferent lymph nodes in EAE mice}

We previously reported that Sirt6 plays a role in DC activation [9]. Sirt6KO BMDDC express lower levels of class II MHC molecules and of key chemokine receptors such as CCR2 as compared to WT BMDDC. The triggering of an adaptive immune response requires DC migration from the site of antigen encounter to the draining lymph nodes [18]. Such migration is typically accomplished through the selective expression of chemokine receptors. Immature DC, which reside in or traffic through peripheral tissue, express defined pattern of chemokine receptors, including CXCR4, while not expressing CCR7, which confers responsiveness to inflammatory stimuli and mediates DC migration to the lymph nodes $[19,20]$. However, upon exposure to inflammatory signals, DC begin their maturation process, downregulating most chemokine receptors with the exception of CXCR4, while upregulating CCR7 [19, 21]. To define whether Sirt6 inhibition also affects DC function, we quantified the representation of DC in lymph nodes isolated from EAE mice that were treated with or without 1 and also measured CCR7 and CXCR4 expression on them by FACS (Fig. 3a, d, g and Additional file 1).

The percentage of $\mathrm{CD} 11 \mathrm{c}^{+} \mathrm{DC}$ in lymph nodes from 1-treated mice was slightly, although not significantly, higher than in vehicle-treated mice at $7 \mathrm{dpi}$ (Fig. 3b). DC activation was significantly reduced by treatment with $\mathbf{1}$ : specifically, the expression of CD40 (a DC activation marker, which is crucial for the early activation of naïve
$\mathrm{T}$ cells, ref. [22]) was decreased in 1-treated mice at 7 dpi (Fig. 3c). Percentages of $\mathrm{CXCR}^{+}$and of $\mathrm{CXCR}^{+} /$ $\mathrm{CCR}^{+}$double-positive $\left(\mathrm{CD} 11 \mathrm{c}^{+}\right) \mathrm{DC}$ were significantly decreased in 1-treated animals, whereas the percentage of $\mathrm{CCR} 7^{+}$CDs cells was only slightly reduced by treatment with 1 (Fig. 3d). At the time of disease onset, in the lymph nodes, the total number of $\mathrm{CD} 11 \mathrm{c}^{+} \mathrm{DC}$, as well as the percentage of activated DC (as assessed by the level of CD86, a costimulatory molecule which is crucial for activating $\mathrm{T}$ cells, ref. [23]) was not significantly different between 1-treated and control animals (Fig. 3e, f). However, the percentage of $\mathrm{CXCR}^{+}$and of $\mathrm{CXCR}^{+} / \mathrm{CCR}^{+} \mathrm{DC}$ was higher in $\mathbf{1}$-treated mice as compared to control animals (Fig. 3g). Altogether, these data suggest that Sirt6 inhibition results in a delay in DC activation and migration: in vehicle-treated mice, $\mathrm{DC}$ are activated at $7 \mathrm{dpi}$, whereas in 1-treated mice, $\mathrm{DC}$ appear to be activated with approximately 5 days of delay (on the day of disease onset in vehicle-treated animals). These findings are in line with the effect of $\mathbf{1}$ on EAE onset when the compound is used as a preventive treatment (Fig. 1d).

\section{Pharmacological SIRT6 inhibition hampers DC migration} As mentioned above, a role for SIRT6 in mature DC migration was previously reported based on findings with DC isolated from wild-type and from Sirt6KO mice [9]. Here, we pre-emptively incubated BMDDC from wildtype animals with or without $\mathbf{1}$ and verified Sirt6 inhibition by monitoring H3K9 acetylation by Western blotting (Fig. 4a). Next, BMDDC generated from wildtype animals and seeded in a 6-well plate, were incubated with or without $\mathbf{1}$, and stimulated to mature with LPS. Cells were recovered at the end of the incubation period. The number of viable cells was not significantly affected by the incubation with compound $1[(3.1 \pm 0.5) \times$ $10^{5}$ and $(2.5 \pm 0.5) \times 10^{5}$ cells/well were recovered from control and compound 1-treated cells, respectively, $n=3$ ]. DC were then challenged to migrate towards a CCL19CCL21 cocktail (which stimulates CCR7) or towards SDF1 (which activates CXCR4). As shown in Fig. 4, cell migration was almost completely abolished in the presence of $\mathbf{1}$, demonstrating that pharmacological Sirt6 inhibition blocks DC migration. These results are in line with our previous findings in DC from Sirt6 KO mice [9].

\section{Sirt6 inhibition promotes an anti-inflammatory cytokine profile}

Given the role of Sirt6 in regulating TNF $\alpha$ expression and release [2-5], we proceeded to measure TNF $\alpha$ concentration in mouse plasma. The levels of TNF $\alpha$ were significantly decreased in animals treated with the SIRT6 inhibitor, 1, both at $7 \mathrm{dpi}$ and at $15-17 \mathrm{dpi}$, i.e., at disease onset in vehicle-treated mice (Fig. 5a). We also evaluated the level of IFN $\gamma$ and of IL12, two cytokines 


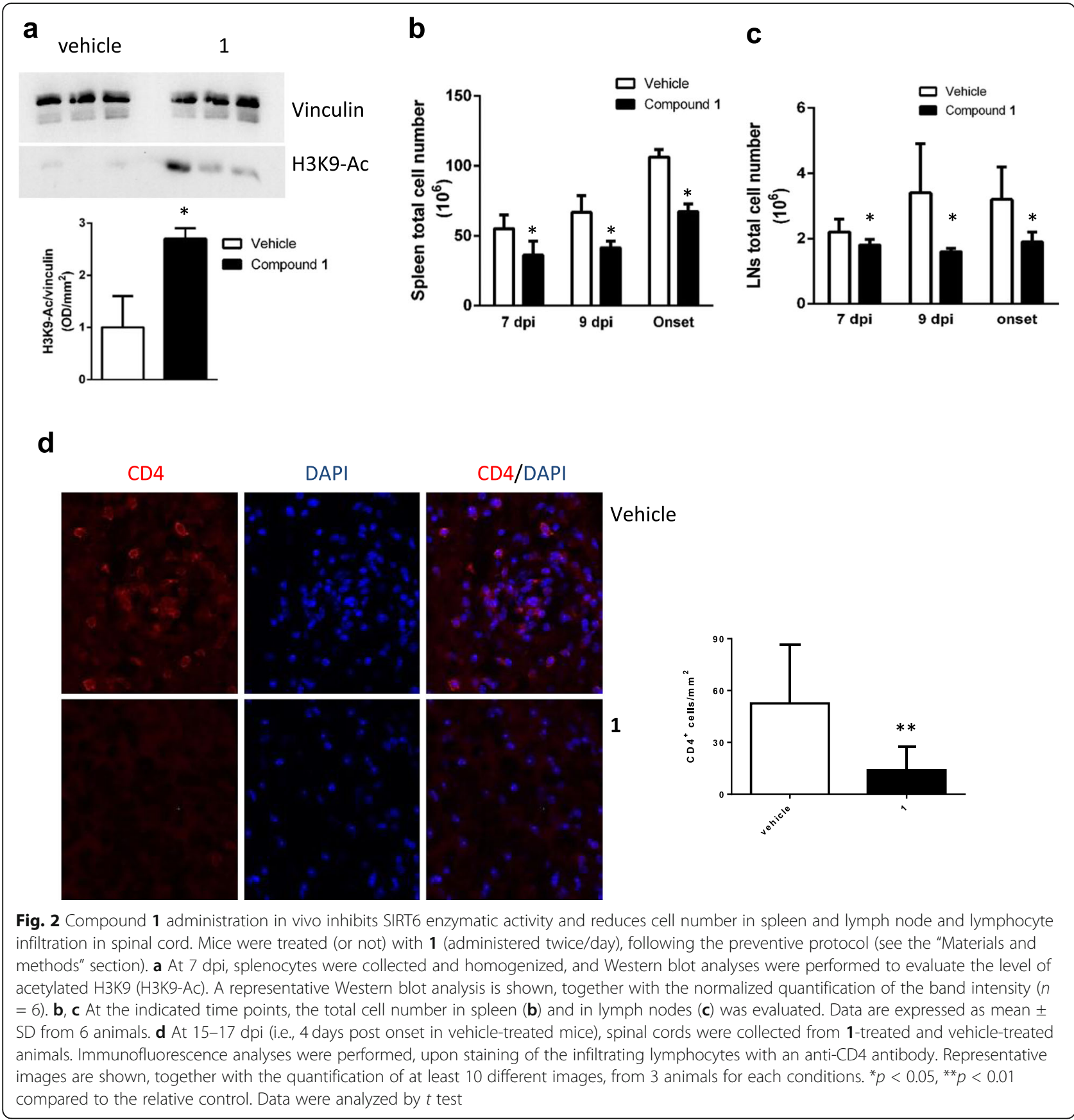

that promote Th1 immune responses, by qPCR on spinal cords collected at EAE onset and found that their expression was also reduced in mice which were given 1 (Fig. 5b). Finally, the expression of IL10, which has antiinflammatory effects, was significantly increased in the spinal cord of 1-treated mice (Fig. 5b). IFNy, IL12, and IL10 expression values were normalized against the housekeeping TBP and HPRT1 genes, since their expression, as well as the expression of $\beta$-actin, were not different in spinal cords from animals treated with $\mathbf{1}$ or with the vehicle (Fig. 5c). Altogether, these data confirm that the delay in EAE onset via treatment with the Sirt6 inhibitor, 1, likely reflects a delayed activation of the immune response.

\section{Discussion}

Both cells of the adaptive and of the innate immune system are involved in several deleterious steps of autoimmune responses, including activation of T cells by DC. EAE is a complex model in which the interaction between a variety of immune system components and the CNS leads to pathological features that are very similar to those of MS: 
a

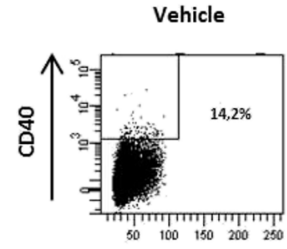

Compound 1
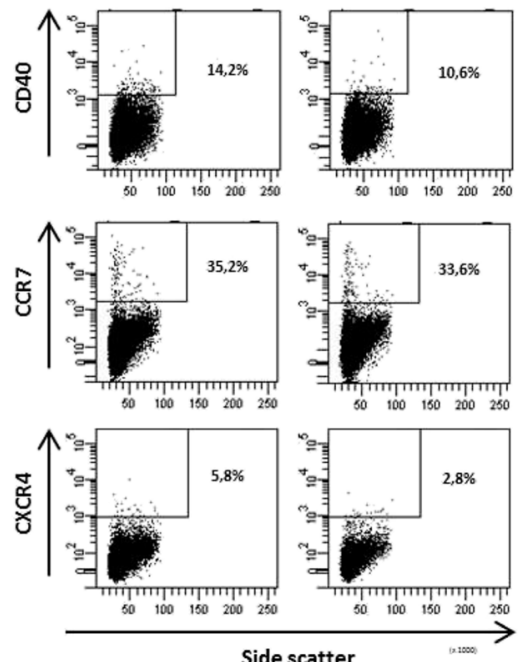

Side scatter

b

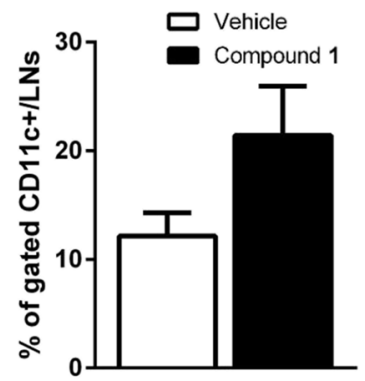

C

CD40

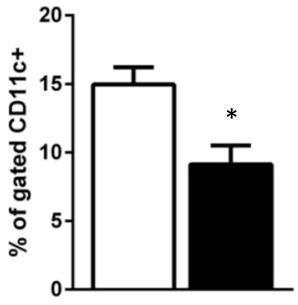

f

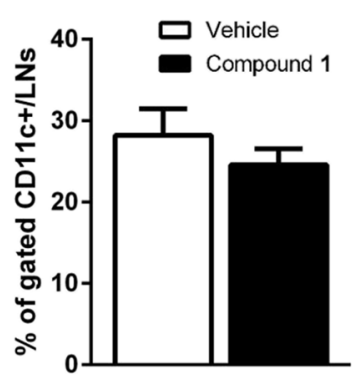

d



g

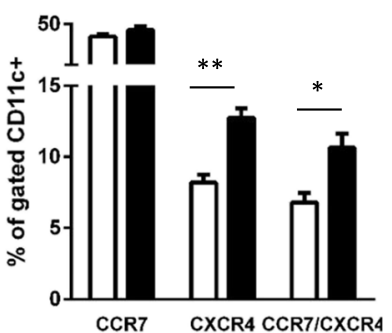

Fig. 3 Sirt6 inhibition determines a delayed influx of CXCR4 ${ }^{+}$DC in lymph nodes of EAE-affected mice. Mice were treated, or not, following the preventive protocol (i.p. administration of $30 \mathrm{mg} / \mathrm{kg} \mathrm{1}$, twice/day). a One representative plot of CD11 $\mathrm{c}^{+} \mathrm{DC}$ stained CD40, CCR7, and CXCR4 is shown. b-d DC were obtained from lymph nodes (LNs) at 7 dpi and stained with antibodies against CD11c (b), or against CD11C and CD40 (c), or against CD11C and CXCR4 and CCR7 (d) and analyzed by FACS. Data are shown as mean \pm SEM. ${ }^{* *} p<0.01$ ( $n=6$ from two independent experiments). e- $\mathbf{g}$ DC were obtained from LNs at 10-13 dpi, i.e., at disease onset in vehicle-treated mice. Cells were stained with antibodies against CD11C (e) or against CD11C and CD86 (f), or against CD11C and CXCR4 and CCR7 (g). Data are shown as mean \pm SEM. ${ }^{*} p<0.05,{ }^{* *} p<$ 0.01 ( $n=6$ from two independent experiments). Data were analyzed by $t$ test

inflammation, demyelination, axonal loss, and gliosis [24]. The adjuvant that is used in EAE to stimulate peripheral immune cells contains killed Mycobacterium tuberculosis, which acts as danger signal: both MOG35-55 and the adjuvant are injected subcutaneously to stimulate local DC that subsequently migrate to the draining lymph nodes to stimulate antigen-specific T cells. After successful activation, encephalitogenic $\mathrm{T}$ cells migrate into the CNS to cause tissue damage. Thus, EAE is used as a model of cell-mediated organ-specific autoimmunity. Our goal was to determine the effect of pharmacological Sirt6 inhibition in DC in EAE as a model of autoimmune disorders. 




SIRT6 plays critical roles in multiple molecular pathways, including DNA repair, telomere maintenance, glycolysis, gluconeogenesis, lipid metabolism, and inflammation [25]. Different attempts to modulate its activity have been investigated as a means to provide therapeutic benefits in different conditions. Mainly in view of its role in DNA repair and in telomere biology and as a tumor suppressor in different cancer type,

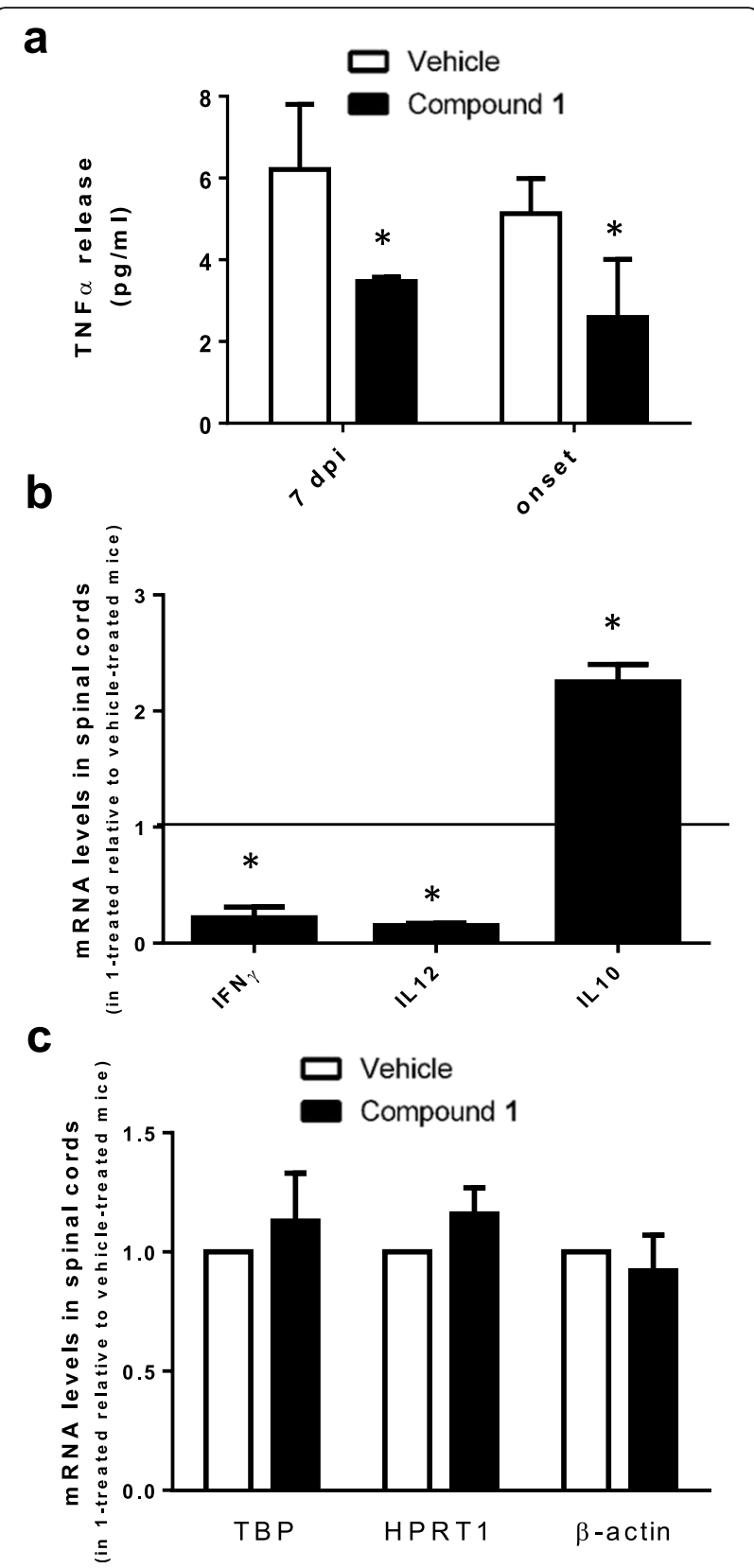

Fig. 5 Effect of compound $\mathbf{1}$ on IFNY, IL12, and IL10 profile in EAE. Mice were treated (or not) with $\mathbf{1}$ (administered twice/day), following the preventive protocol (see the "Materials and methods" section). a At 7 dpi and at 13-17 dpi (i.e., onset for vehicle-treated mice), mice were sacrificed and TNFa was evaluated in the collected plasma. b, c Spinal cords were collected at 13-17 dpi (i.e., onset for vehicle-treated mice), and the relative expression of IFNY, IL12, and IL10 (b) and of TBP, HPRT1, and $\beta$-actin (used as "housekeeping" genes) (c) was analyzed by qPCR analysis. Samples from at least 4 animals were analyzed for each condition. ${ }^{*} p<0.05$, compared to controls (data analyzed by $t$ test)

activation of SIRT6 is postulated to have beneficial effects and to promote healthspan [25]. Nevertheless, the development of SIRT6 inhibitors has also been pursued 
as these compounds could find application in some cancer types, in which SIRT6 seems to have pro-oncogenic effects (i.e., squamous cell carcinomas, pancreatic cancer, acute myeloid leukemia, and multiple myeloma), such as increasing cell migration, tumor DNA repair, and secretion of pro-angiogenic factors [5, 13, 26, 27]. In addition, given the fact that SIRT6 regulates insulin signaling [28] and the expression of the glucose transporters GLUT1 and GLUT4 [7, 28], we previously tested $\mathbf{1}$ in a mouse model of type 2 diabetes. Here we found different metabolic parameters to be improved by $\mathbf{1}$, including glycemia, insulinemia, triglycerides, and cholesterol, following activation of glucose consumption [14]. In addition, enhancement of glycolysis via SIRT6 inhibition was shown to promote survival of photoreceptor and to preserve vision in a mouse model of retinitis pigmentosa [29]. Concerning neurodegenerative and inflammatory diseases, SIRT6 inhibition was proposed to be protective at least in certain circumstances [30]. For instance, inhibiting SIRT6 was suggested as a promising strategy against Parkinson's disease, given that SIRT6 has pro-inflammatory effects in this disorder and thereby accelerates its course [30].

As reported in the "Introduction" section, SIRT6 plays a multifaceted role in the regulation of the immunity, which can be summarized as follows: (a) SIRT6 promotes the release of TNF $\alpha$ from different cells (including DC) through different mechanisms [2-4, 9]; (b) SIRT6 promotes the secretion of IFN $\gamma$ and of IL8 [3, 5, 6]; and (c) SIRT6 enhances DC differentiation and maturation [9].

Thus, based on SIRT6 role in inflammation and in DC, we decided to test the SIRT6 inhibitor, 1, in the EAE model of autoimmunity according to both a "preventive" and a "therapeutic" protocol. The most interesting result from this study was the delay in EAE onset, which was obtained by inhibiting Sirt6 according to our "preventive" approach. By this approach, we aimed at investigating the effect of Sirt6 inhibition during the early phase of the inflammatory process preceding the overt disease. In line with the studies indicating SIRT6 as a key player in regulating proinflammatory responses, our results indicate that SIRT6 inhibition delayed the inflammatory cascade occurring upon immunization and before EAE onset. Specifically, SIRT6 inhibition with 1 reduced the number of splenocytes and of lymph node cells in mice (Fig. 2b, c); blunted the counts of lymphocytes infiltrating the spinal cord (Fig. 2d); reduced the production of the autoimmunity-promoting cytokines, IFNY and IL12 (Fig. 5); and increased the production of the anti-inflammatory cytokine, IL-10 (Fig. 5). In addition, 1 reduced the percentage of mouse lymph node DC expressing the pro-migratory surface marker, CXCR4 (Fig. 3).

Given the well-established role of peripheral and CNSresident DC in the development of EAE [31, 32], we propose that the delay in the onset of EAE upon mouse treatment with 1 reflects the effect of Sirt6 inhibition on
DC migration, as revealed by the reduced representation of CXCR4-positive and of CXCR4/CCR7-double-positive DC in lymph nodes [33] of 1-treated animals (Fig. 3) and by the impaired migration of 1-pre-treated DC in response to chemokines (Fig. 4). The mechanism by which Sirt6 inhibition reduced DC migration in vivo might also reflect the fact that TNF $\alpha$ release (as measured by evaluating TNF $\alpha$ in plasma, Fig. 5a) was reduced upon SIRT6 inhibition, which is in line with the reported ability of SIRT6 to regulate TNF $\alpha$ production by different cell types [2-5]. Indeed, TNF $\alpha$ is a crucial cytokine in DC activation and migration [34, 35].

Overall, our results provide a rationale for further exploring SIRT6 inhibition as a strategy to treat MS or other autoimmune disorders by virtue of its effects on the DC compartment. Given the fact that Sirt6 inhibition failed to show an impact on disease course in the therapeutic protocol (where 1 administration was started at disease onset; see Fig. 1 and the "Results" section), SIRT6 is unlikely to represent a viable target for treating the overt MS. Nevertheless, SIRT6 inhibition may be tested during the early stages of an immune-mediated condition to prevent exacerbations and progression of the disease. For instance, the so-called clinically isolated syndrome (CIS) is often the first indicator of MS, with few available therapeutic options to avoid its progression to overt MS [36]. As compared with healthy subjects, patients with CIS exhibit a peripheral blood signature that is characterized by a higher frequency of DC, and this suggest a role of these antigenpresenting cells in CIS conversion to MS [37]. Thus, early treatment of these patients with a SIRT6 inhibitor could conceivably avoid or at least slow the CIS progression to MS, due to the effect exerted on DC migration.

\section{Conclusions}

The present study demonstrates that preventive pharmacological SIRT6 inhibition delayed EAE disease onset, reducing the representation of CXCR4-positive and of CXCR4/CCR7double-positive DC in lymph nodes. The delay in EAE onset was associated with the early downregulation of CD40 expression on DC and with reduced encephalitogenic $\mathrm{T}$ cell infiltration in the CNS. Sirt6 inhibition reduced the production of the autoimmunity-promoting cytokines, IFN $\gamma$ and IL12, and increased the production of the anti-inflammatory cytokine IL-10. Therefore, Sirt6 might represent a valuable target for developing novel therapeutic agents for the treatment of the early stages of MS or of other autoimmune disorders. The administration of SIRT6 inhibitors should be done with caution, limiting it to defined time windows, taking into account that this enzyme also has a role as a tumor suppressor in certain tissues and that complete SIRT6 depletion can be very detrimental [7, 23]. Nevertheless, as discussed above, growing evidence does point to SIRT6 inhibition as to a promising strategy in defined conditions, including MS. 


\section{Supplementary information}

Supplementary information accompanies this paper at https://doi.org/10. 1186/s12974-020-01906-1.

Additional file 1:. Figure S1. Gating strategy for flow cytometry analysis of DCs

\section{Abbreviations}

SIRT6: Sirtuin 6; 2,4-dioxo-N-(4-(pyridin-3 yloxy)phenyl)-1,2,3,4tetrahydroquinazoline-6-sulfonamide (SIRT6 inhibitor), 1; DC: dendritic cells; EAE: experimental autoimmune encephalomyelitis; MOG: myelin oligodendrocyte glycoprotein; dpi: days post immunization; dpo: days post onset; LNs: lymph nodes; CXCR4: C-X-C chemokine receptor type 4; CCR7: CC chemokine receptor type 7; MS: multiple sclerosis; CIS: clinically isolated syndrome

\section{Acknowledgements}

We thank Dr Nicole Kerlero de Rosbo (DINOGMI, University of Genova, Italy) for her helpful suggestions and critical reading of our manuscript. We are indebted with Prof. Emanuela Marcenaro (DIMES, University of Genova, Italy) for providing useful reagents.

\section{Authors' contributions}

GF designed a part of the research, planned and performed the experiments, analyzed the data, and collaborated in drafting the manuscript; $A B$ performed the experiments and analyzed the data; LS, DM, DF, SS, EA, MvH, and MM performed the experiments; AN designed the research and revised the manuscript; $\mathrm{AU}$ analyzed the data and revised the manuscript; SB designed the whole research project, analyzed the data, and wrote the manuscript. The authors read and approved the final manuscript.

\section{Funding}

This work was also supported by FISM - Fondazione Italiana Sclerosi Multipla - cod. 2017/R/6 and financed or co-financed with the "5 per mille" public funding (to S.B.)

\section{Availability of data and materials}

The datasets used and/or analyzed during the current study are available from the corresponding author on reasonable request.

\section{Ethics approval and consent to participate}

We declare that we obtained the ethics approval by the Ethical Committee for Animal Experimentation of the IRCCS San Martino hospital, Genoa, Italy, and by the Italian Ministry of Health (project nr, 553/2018-PR).

\section{Consent for publication}

Not applicable.

\section{Competing interests}

We declare that all co-authors have no competing interests for this study.

\section{Author details}

1Ospedale Policlinico San Martino, IRCCS, Largo R. Benzi, 10, 16132 Genova, Italy. ${ }^{2}$ Department of Experimental Medicine (DIMES), University of Genova, Genova, Italy. ${ }^{3}$ Department of Neuroscience, Rehabilitation, Ophthalmology, Genetics, Maternal and Child Health (DINOGMI), University of Genova, Genova, Italy. ${ }^{4}$ Roche Diagnostics International AG, Rotkreuz, Switzerland. ${ }^{5}$ Cellestia Biotech AG, Basel, Switzerland. ${ }^{6}$ Department of Internal Medicine and Medical Specialties (DIMI), University of Genova, Genova, Italy.

Received: 27 April 2020 Accepted: 20 July 2020

Published online: 31 July 2020

\section{References}

1. Kugel S, Mostoslavsky R. Chromatin and beyond: the multitasking roles for SIRT6. Trends Biochem Sci. 2014;39(2):72-81.

2. Van Gool F, Gallí M, Gueydan C, Kruys V, Prevot PP, Bedalov A, et al. Intracellular NAD levels regulate tumor necrosis factor protein synthesis in a sirtuin-dependent manner. Nat Med. 2009;15(2):206-10.
3. Bruzzone S, Fruscione F, Morando S, Ferrando T, Poggi A, Garuti A, et al. Catastrophic NAD ${ }^{+}$depletion in activated T lymphocytes through Nampt inhibition reduces demyelination and disability in EAE. PLoS One. 2009;4(11): e7897.

4. Jiang H, Khan S, Wang Y, Charron G, He B, Sebastian C, et al. SIRT6 regulates TNF-a secretion through hydrolysis of long-chain fatty acyl lysine. Nature. 2013;496(7443):110-3.

5. Bauer I, Grozio A, Lasigliè D, Basile G, Sturla L, Magnone M, et al. The NAD+_ dependent histone deacetylase SIRT6 promotes cytokine production and migration in pancreatic cancer cells by regulating Ca2+ responses. J Biol Chem. 2012;287(49):40924-37.

6. Montecucco F, Bauer I, Braunersreuther V, Bruzzone S, Akhmedov A, Lüscher TF, et al. Inhibition of nicotinamide phosphoribosyltransferase reduces neutrophil-mediated injury in myocardial infarction. Antioxid Redox Signal. 2013:18(6):630-41.

7. Mostoslavsky R, Chua KF, Lombard DB, Pang WW, Fischer MR, Gellon L, et al. Genomic instability and aging-like phenotype in the absence of mammalian SIRT6. Cell. 2006;124(2):315-29.

8. Liu TF, Vachharajani VT, Yoza BK, McCall CE. NAD+-dependent sirtuin 1 and 6 proteins coordinate a switch from glucose to fatty acid oxidation during the acute inflammatory response. J Biol Chem. 2012;287(31):25758-69.

9. Lasigliè D, Boero S, Bauer I, Morando S, Damonte P, Cea M, et al. Sirt6 regulates dendritic cell differentiation, maturation, and function. Aging (Albany NY). 2016;8(1):34-49.

10. Parenti MD, Grozio A, Bauer I, Galeno L, Damonte P, Millo E, et al. Discovery of novel and selective SIRT6 inhibitors. J Med Chem. 2014;57(11):4796-804.

11. Sociali G, Galeno L, Parenti MD, Grozio A, Bauer I, Passalacqua M, et al. Quinazolinedione SIRT6 inhibitors sensitize cancer cells to chemotherapeutics. Eur J Med Chem. 2015;102:530-9.

12. Damonte P, Sociali G, Parenti MD, Soncini D, Bauer I, Boero S, et al. SIRT6 inhibitors with salicylate-like structure show immunosuppressive and chemosensitizing effects. Bioorg Med Chem. 2017;25(20):5849-58.

13. Cagnetta A, Soncini D, Orecchioni S, Talarico G, Minetto P, Guolo F, et al. Depletion of SIRT6 enzymatic activity increases acute myeloid leukemia cells' vulnerability to DNA-damaging agents. Haematologica. 2018;103(1):80-90.

14. Sociali G, Magnone M, Ravera S, Damonte P, Vigliarolo T, Von Holtey M, et al. Pharmacological Sirt6 inhibition improves glucose tolerance in a type 2 diabetes mouse model. FASEB J. 2017:31(7):3138-49.

15. Ferrara G, Errede M, Girolamo F, Morando S, Ivaldi F, Panini N, et al. NG2, a common denominator for neuroinflammation, blood-brain barrier alteration, and oligodendrocyte precursor response in EAE, plays a role in dendritic cell activation. Acta Neuropathol. 2016;132(1):23-42.

16. Livak J, Schmittgen TD. Analysis of relative gene expression data using realtime quantitative $P C R$ and the 2- $\Delta \Delta C T$ method. Methods. 2001:25(4):402-8.

17. Bruzzone S, Moreschi I, Usai C, Guida L, Damonte G, Salis A, et al. Abscisic acid is an endogenous cytokine in human granulocytes with cyclic ADP-ribose as second messenger. Proc Natl Acad Sci U S A. 2007;104(14):5759-64.

18. Ricart BG, John B, Lee D, Hunter CA, Hammer DA. Dendritic cells distinguish individual chemokine signals through CCR7 and CXCR4. J Immunol. 2011; 186(1):53-61.

19. De Smedt T, Pajak B, Muraille E, Lespagnard L, Heinen E, De Baetselier P et al. Regulation of dendritic cell numbers and maturation by lipopolysaccharide in vivo. J Exp Med. 1996:184(4):1413-24.

20. Sallusto F, Palermo B, Lenig D, Miettinen M, Matikainen S, Julkunen I, et al. Distinct patterns and kinetics of chemokine production regulate dendritic cell function. Eur J Immunol. 1999;29(5):1617-25.

21. Yanagihara S, Komura E, Nagafune J, Watarai H, Yamaguchi Y. EBI1/CCR7 is a new member of dendritic cell chemokine receptor that is up-regulated upon maturation. J Immunol. 1998;161(6):3096-102.

22. Haase C, Michelsen BK, Jørgensen TN. CD40 is necessary for activation of naïve $T$ cells by a dendritic cell line in vivo but not in vitro. Scand J Immunol. 2004;59(3):237-45.

23. Dilioglou S, Cruse JM, Lewis RE. Function of CD80 and CD86 on monocyteand stem cell-derived dendritic cells. Exp Mol Pathol. 2003;75(3):217-27.

24. Gold R, Linington C, Lassmann $\mathrm{H}$. Understanding pathogenesis and therapy of multiple sclerosis via animal models: 70 years of merits and culprits in experimental autoimmune encephalomyelitis research. Brain. 2006;129(Pt 8): 1953-71.

25. Chang AR, Ferrer CM, Mostoslavsky R. SIRT6, a mammalian deacylase with multitasking abilities. Physiol Rev. 2020;100(1):145-69. 
26. Lefort K, Brooks Y, Ostano P, Cario-Andre M, Calpini V, Guinea-Viniegra J, et al. A miR-34a SIRT6 axis in the squamous cell differentiation network. EMBO J. 2013;32(16):2248-63.

27. Liu Y, Xie QR, Wang B, Shao J, Zhang T, Liu T, et al. Inhibition of SIRT6 in prostate cancer reduces cell viability and increases sensitivity to chemotherapeutics. Protein Cell. 2013;4(9):702-10.

28. Xiao C, Kim HS, Lahusen T, Wang RH, Xu X, Gavrilova O, et al. SIRT6 deficiency results in severe hypoglycemia by enhancing both basal and insulin stimulated glucose uptake in mice. J Biol Chem. 2010;285(47):36776-84.

29. Zhang L, Du J, Justus S, Hsu CW, Bonet-Ponce L, Wu WH, et al. Reprogramming metabolism by targeting sirtuin 6 attenuates retinal degeneration. J Clin Invest. 2016;126(12):4659-73.

30. Nicholatos JW, Francisco AB, Bender CA, Yeh T, Lugay FJ, Salazar JE, et al. Nicotine promotes neuron survival and partially protects from Parkinson's disease by suppressing SIRT6. Acta Neuropathol Commun. 2018:6(1):120.

31. Mohammad MG, Hassanpour M, Tsai WW, Li H, Ruitenberg MJ, Booth DW, et al. Dendritic cells and multiple sclerosis: disease, tolerance and therapy. Int J Mol Sci. 2012;14(1):547-62.

32. Zozulya AL, Clarkson BD, Ortler S, Fabry Z, Wiendl H. The role of dendritic cells in CNS autoimmunity. J Mol Med (Berl). 2010;88(6):535-44.

33. Butcher EC, Picker L. Lymphocyte homing and homeostasis. Science. 1996; 272(5258):60-6.

34. Trevejo JM, Marino MW, Philpott N, Josien R, Richards EC, Elkon KB, et al. TNF-alpha -dependent maturation of local dendritic cells is critical for activating the adaptive immune response to virus infection. Proc Natl Acad Sci U S A. 2001:98(21):12162-7.

35. Ebrahimi M, Hassan ZM, Hadjati J, Hayat P, Moazzeni SM. Immediate exposure to TNF-alpha activate dendritic cells derived from non-purified cord blood mononuclear cells. Iran J Immunol. 2009;6(3):107-18.

36. Grigoriadis N, van Pesch V; Paradig MS Group. A basic overview of multiple sclerosis immunopathology. Eur J Neurol. 2015;22 Suppl 2:3-13.

37. Trend S, Jones AP, Geldenhuys S, Byrne SN, Fabis-Pedrini MJ, Nolan D, et al. Evolving identification of blood cells associated with clinically isolated syndrome: importance of time since clinical presentation and diagnostic MRI. Int J Mol Sci. 2017:18(6)

\section{Publisher's Note}

Springer Nature remains neutral with regard to jurisdictional claims in published maps and institutional affiliations.

Ready to submit your research? Choose BMC and benefit from:

- fast, convenient online submission

- thorough peer review by experienced researchers in your field

- rapid publication on acceptance

- support for research data, including large and complex data types

- gold Open Access which fosters wider collaboration and increased citations

- maximum visibility for your research: over $100 \mathrm{M}$ website views per year

At $\mathrm{BMC}$, research is always in progress.

Learn more biomedcentral.com/submissions 